TITLE:

\title{
Structure and dynamics of transient gels
}

$\operatorname{AUTHOR}(\mathrm{S})$ :

Tanaka, Fumihiko

CITATION:

Tanaka, Fumihiko. Structure and dynamics of transient gels. Progress of Theoretical Physics Supplement 1997, 126: 257-260

ISSUE DATE:

1997

URL:

http://hdl.handle.net/2433/49183

RIGHT:

Copyright (c) Progress of Theoretical Physics 1997 All rights reserved. 


\title{
Structure and Dynamics of Transient Gels
}

\author{
Fumihiko TANAKA*) \\ Department of Applied Physics, Tokyo University of Agriculture and Technology \\ Koganei-shi, Tokyo 184, Japan
}

\begin{abstract}
A simple transient network model is introduced to describe creation and annihilation of the junctions in the networks of associating polymers. The time-evolution equation under arbitrary deformation is derived for the number of elastically effective polymer chains in the network. It is found that rheological properties depend rather sensitively on the chain disengagement rate $\beta(\boldsymbol{r})$ and recombination probability $p$ of associative groups. The dynamic mechanical moduli are calculated as functions of the frequency $\omega$ and the chain disengagement rate. From the peak of the loss modulus, the lifetime $\tau_{\times}$of the junction is estimated, and from the high frequency plateau of the storage modulus the number of elastically effective chains in the network is found. On the basis of the Scanlan-Case criterion, the number of elastically effective chains and the number of dangling ends are calculated as functions of the polymer concentration and the temperature. The results are compared with the recent experimental report on the elastic moduli of hydrophobically modified water-soluble polymers.
\end{abstract}

\section{§1. Introduction -Model transient network-}

Most thermoreversible gels have multiple junctions combining more than two network chains. The number of chains bound together in a single junction is called multiplicity of the junction. Multiple junctions formed by weak associative interaction can break and recombine under thermal fluctuation and/or external stress with a finite average lifetime $\tau_{\times}$. To describe creation and annihilation of the junctions, we consider a model network made up of polymers of uniform molecular weight $M$ (or the number $n$ of statistical units) carrying associative functional groups at their both chain ends (named telechelic polymer). ${ }^{1)}$ We focus our attention specifically on the unentangled regime where $M$ is smaller than the entanglement molecular weight $M_{e}$, so that each chain obeys Rouse dynamics modified by mutual end-association. In the following, we assume the lifetime of a junction is sufficiently long so that it is well separated from the Rouse relaxation spectrum, the longest time of which is given by $\tau_{R} \equiv \zeta a^{2} n^{2} / 3 \pi^{2} k T$, where $\zeta$ is the friction coefficient of a monomer.

We consider a time interval $d t$ smaller than $\tau_{\times}$but still larger than $\tau_{R}$. Under a macroscopic deformation $\hat{\lambda}(t)$ given to the network, either end of a chain, being stretched above a critical length, disengages from the junction and the chain relaxes to a Gaussian conformation, whilst some of the free dangling ends recapture the junctions in their neighborhood (Fig. 1). Since the stress is transmitted only through the chains whose both ends are connected to the network junctions, we call these chains elastically effective (or active) chains.

Let $\nu_{0}$ be the total number of polymer chains in a unit volume, and let $F(\boldsymbol{r}, t) d \boldsymbol{r}$

*) Present address: Department of Polymer Chemistry, Graduate School of Engineering, Kyoto University, Kyoto 606-01, Japan. 
be the number of active chains at time $t$ whose end-to-end vector $r$ falls onto a small region $d \boldsymbol{r}$. The total number of active chains at time $t$ is given by $\nu(t) \equiv \int F(\boldsymbol{r}, t) d \boldsymbol{r}$. It is shown, by counting the number of active chains that are destroyed and created in the interval $d t$ under the deformation $\hat{\lambda}(t)$, that the time development of the distribution is governed by the equation

$$
F(\boldsymbol{r}, t) d \boldsymbol{r}=\Theta\left(\boldsymbol{r}, t ; \boldsymbol{r}_{0}, 0\right) F\left(\boldsymbol{r}_{0}, 0\right) d \boldsymbol{r}_{0}+p \int_{0}^{t} d t^{\prime} \Theta\left(\boldsymbol{r}, t ; \boldsymbol{r}^{\prime}, t^{\prime}\right)\left[\nu_{0}-\nu\left(t^{\prime}\right)\right] f_{0}\left(\boldsymbol{r}^{\prime}\right) d \boldsymbol{r}^{\prime},
$$

where $\Theta\left(\boldsymbol{r}, t ; \boldsymbol{r}^{\prime}, t^{\prime}\right) \equiv \exp \left[-\int_{t^{\prime}}^{t} \beta\left(\boldsymbol{r}_{t^{\prime \prime}, t^{\prime}}\right) d t^{\prime \prime}\right]$ is a probability for an active chain created at time $t^{\prime}$ in the past to remain active until the present time $t$. The function $\beta(r)$ gives the probability per unit time for an active chain to disengage from the junction and is called chain breakage rate. The parameter $p$ in the second term is the probability per unit time for a dangling end to capture a junction (called chain recombination rate). We have assumed that end-to-end vectors are mapped affinely to the macroscopic deformation so that we have $\boldsymbol{r}_{t^{\prime \prime}, t^{\prime}}=\hat{\lambda}\left(t^{\prime \prime}\right) \cdot \hat{\lambda}\left(t^{\prime}\right)^{-1} \cdot \boldsymbol{r}^{\prime}$, etc. The equilibrium distribution $f_{0}(r)$ of the end-to-end vector is assumed to take the conventional Gaussian form with the average square distance $\left\langle r^{2}\right\rangle_{0}=n a^{2}$.

\section{§2. Dynamic mechanical moduli}

On the basis of this fundamental equation, we find that the storage modulus $G^{\prime}(\omega, T)$ and the loss modulus $G^{\prime \prime}(\omega, T)$ for shear deformation of the network is given by

$$
\begin{aligned}
& G^{\prime}(\omega, T)=\nu_{e}(T) k T g_{1}(\omega, T), \\
& G^{\prime \prime}(\omega, T)=\nu_{e}(T) k T g_{2}(\omega, T),
\end{aligned}
$$

where $\nu_{e}(T)$ is the number of elastically effective chains in a unit volume of the network at temperature $T$, and the new functions $g_{i}(\omega)$ are explicitly given by

$$
\begin{gathered}
g_{1}(\omega) \equiv \frac{1}{\zeta_{0}\left\langle r^{2}\right\rangle_{0}}\left\langle\frac{\omega^{2} r^{2}}{\beta(r)\left[\omega^{2}+\beta(r)^{2}\right]}\left[1-\frac{2 r \beta(r) \beta^{\prime}(r)}{5\left[\omega^{2}+\beta(r)^{2}\right]}\right]\right\rangle_{0}, \\
g_{2}(\omega) \equiv \frac{1}{\zeta_{0}\left\langle r^{2}\right\rangle_{0}}\left\langle\frac{\omega r^{2}}{\omega^{2}+\beta(r)^{2}}\left[1+\frac{\left[\omega^{2}-\beta(r)^{2}\right] r \beta^{\prime}(r)}{5 \beta(r)^{2}\left[\omega^{2}+\beta(r)^{2}\right]}\right]\right\rangle_{0} .
\end{gathered}
$$

In the extreme limit where the chain breakage rate $\beta(r)$ is given by a constant value $\beta_{0}$, our model is reduced to the conventional Green-Tobolsky equation. To find more realistic form of the chain breakage rate, let us consider that the free energy barrier for an associative group to disengage from a junction is given by $W$. Since the tension $f=\left(3 k T / n a^{2}\right) r$ along the effective chain with end-to-end distance $r$ reduces this barrier to $W-f \cdot a$, the chain breakage rate $\beta(r)$ is expected to be proportional to $\exp [-(W-f \cdot a)]$. We thus find $\beta(r)$ takes the form

$$
\beta(r)=\beta_{0} e^{\kappa r},
$$


where $\beta_{0}(T) \equiv \omega_{0} \exp (-W / k T)$ is the reciprocal of the duration time $\tau_{\times}$of a bond ( $\omega_{0}$ being the intrinsic frequency of the vibration of the group), and $\kappa \equiv 3 / n a$ a constant depending on the molecular weight of the chain.

Upon substitution of $(2 \cdot 5)$ into the moduli, we find that a modulus-frequency curve at any temperature $T$ can be superimposed onto a single curve at the reference temperature $T_{0}$, if it is horizontally and vertically shifted properly. Specifically we have

$$
\frac{G(\omega)}{\nu_{e}\left(T_{0}\right) k T_{0}} b_{T}=g\left(\frac{\omega}{\beta_{0}\left(T_{0}\right)} a_{T}\right)
$$

for both $G^{\prime}$ and $G^{\prime \prime}$, where

$$
\begin{aligned}
a_{T} & \equiv \beta_{0}\left(T_{0}\right) / \beta(T) \\
& =\exp \left[-\frac{W}{k}\left(\frac{1}{T_{0}}-\frac{1}{T}\right)\right]
\end{aligned}
$$

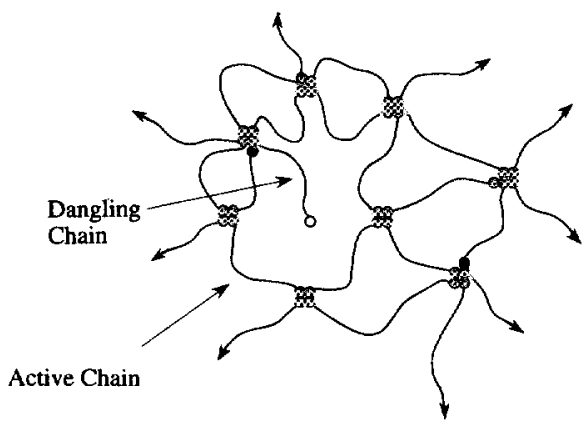

DEFORMATION

is the frequency (horizontal) shift factor, and

$$
b_{T} \equiv \nu_{e}\left(T_{0}\right) k T_{0} / \nu_{e}(T) k T
$$

is the modulus (vertical) shift factor. From $a_{T}$ or the peak position of the loss modulus we find the free energy barrier $W$ of activation, and from $b_{T}$ or the high frequency plateau value of the storage modulus we find the number $\nu_{e}$ of elastically effective chains.

\section{§3. Structure of the transient networks}

To count the number $\nu_{e}$ of elastically effective chains, let us next specify the type of junctions in more detail. A junction of multiplicity $k$ that is connected to the matrix of the gel network through $i$ paths is referred to as an $(i, k)$-junction. Let $\mu_{i, k}$ be the number of junctions specified by the type $(i, k)$ for $k=1,2,3,4, \cdots$ and for $0 \leq i \leq 2 k$.

We now employ the criterion of Scanlan ${ }^{2)}$ and Case ${ }^{3)}$ that only subchains connected at both ends to junctions with at least three paths to the gel are elastically effective. We thus have $i, i^{\prime} \geq 3$ for an effective chain. A junction with one path $(i=1)$ to the gel unites a group of subchains dangling from the network matrix whose conformations are not affected by an applied stress. A junction with two paths $(i=2)$ to the gel merely extends the length of an effective subchain. We may 
call the junctions with $i \geq 3$ elastically effective junctions. An effective subchain is defined as a chain connected to two effective junctions at its both ends. We thus find

$$
\nu_{e}=\frac{1}{2} \sum_{k=2}^{\infty} \sum_{i=3}^{2 k} i \mu_{i, k}
$$

for the number of elastically effective chains.

These results can be compared with the experimental data ${ }^{4)}$ on the high frequency dynamic modulus for HEUR measured by Annable et al. ${ }^{5)}$ Their experimental data for HEUR $\mathrm{C} 16 / 35 \mathrm{~K}$ (end-capped with $\mathrm{C}_{16} \mathrm{H}_{33}$, molecular weight 35,000, Fig. 19 in Ref. 5)) are compared with our theoretical calculation (Fig. 2). Because of the association constant $\lambda(T)$ accompanying the concentration, and also of the difference in the unit of the polymer concentration, we have horizontally shifted the experimental data. Although fitting by a single theoretical curve with a fixed multiplicity is impossible due to the polydispersity in the multiplicity, our theory produces correct behavior over a wide range of the concentration with the multiplicity covering the range from 4 to 7 .

\section{$\S 4$. Conclusions and discussion}

In this study an attempt has been made to find the linear viscoelastic properties of physically cross-linked networks with a finite junction dissociation time $\tau_{\times}$. The model proposed in this study approaches two extreme limits for particular values of $\tau_{\times}$: the covalently crosslinked network $\left(\tau_{\times}=\infty\right)$, and the uncrosslinked melt $\left(\tau_{\times}=0\right)$. The activation barrier for a chain dissociation and the number of elastically effective chains in the network are theoretically estimated and compared with the experiments. This study provides a possible pathway to modelling the new types of transient networks.

\section{References}

1) F. Tanaka and S. F. Edwards, Macromolecules 25 (1992), 1516.

F. Tanaka and S. F. Edwards, J. Non-Newtonian Fluid Mech. 43 (1992), 247, 272, 289.

2) J. Scanlan, J. Polym. Sci. 43 (1960), 501.

3) L. C. Case, J. Polym. Sci. 45 (1960), 397.

4) F. Tanaka and M. Ishida, Macromolecules 29 (1996), 7571.

5) T. Annable, R. Buscall, R. Ettelaie and D. Whittlestone, J. Rheol. 37 (1993), 695.

T. Annable, R. Buscall, R. Ettelaie, P. Shepherd and D. Whittlestone, Langmuir 10 (1994), 1060. 